\title{
Activity types for X-ray candidate AGN from SDSS
}

\section{Gurgen M. Paronyan, Gohar S. Harutyunyan and Areg M. Mickaelian}

Byurakan Astrophysical Observatory (BAO), Byurakan 0213, Aragatzotn province, Armenia Email: gurgen@bao.sci.am, goharutyunyan@gmail.com, aregmick@aras.am

\begin{abstract}
The Joint Catalogue of Hamburg ROSAT Sources (HRC/BHRC) is the result of merging of HRC and BHRC catalogs built on the basis of optical identifications of ROSAT BSC and ROSAT FSC. Altogether, 8132 sources are present. Based on this catalogue, we have compiled a sample of ROSAT AGN, including candidate ones. In this paper we classify candidate AGN (those that previously had not been spectroscopically classified) by their activity type. The sample contains 955 objects with count rate of photons $C R>0.04 \mathrm{ct} / \mathrm{s}$ in the area with galactic latitudes $|b|>30$ and declinations $\delta>0$, however only 217 objects have SDSS DR10 spectra. The classification led to the following results: 95 AGN, 71 absorption-line galaxies, 42 stars, and 9 unclassified objects.
\end{abstract}

Keywords. surveys, cross-correlations, X-ray: AGN, AGN candidates, spectroscopy

By merging Hamburg-ROSAT (HRC, Zickgraf et al. 2003) and Byurakan-HamburgROSAT (BHRC, Mickaelian et al. 2006) catalogs made up on the basis of optical identifications of ROSAT BSC (Voges et al. 1999) and ROSAT FSC (Voges et al. 2000) sources by means of Hamburg Quasar Survey (HQS, Hagen et al. 1995) low-dispersion spectra, we have built the Joint Catalogue of Hamburg ROSAT Sources (HRC/BHRC, Paronyan \& Mickaelian 2014) containing 8132 ROSAT sources. The sample of AGN and AGN candidates consists of 4253 objects, out of which 3352 are found in the Catalogue of QSOs and AGN (Veron-Cetty \& Veron 2010) and Rome Blazar Catalogues (Massaro et al. 2009). There are 2908 objects in the SDSS DR10 (Ahn et al. 2013) giving possibility of their classification, particularly the activity types for AGN. There are 955 candidate AGN, i.e. objects that previously had not been spectroscopically classified. In this paper we classify SDSS spectra of these candidate AGN by their activity type, a smaple with count rate of X-ray photons $C R>0.04 \mathrm{ct} / \mathrm{s}$ in the area with galactic latitudes $|b|>30$ and declinations $\delta>0$. Only 217 objects out of 955 have SDSS DR10 spectra.

Table 1. Results of the spectral classification of ROSAT sources based on SDSS spectra.

\begin{tabular}{|l|r||r|r|}
\hline Active galaxies & Number & Stars & Number \\
\hline Quasars & 5 & subdwarfs (sd) & 1 \\
S1.2 & 2 & white dwarfs (WD) & 4 \\
S1.5 & 15 & cataclysmic variables (CV) & 7 \\
NLS1.5 & 12 & carbon (C) stars & 3 \\
S1.8 & 8 & Other (bright) stars & 26 \\
S1.9 & 6 & & \\
HII regions & 29 & & \\
AGN without definite type & 5 & & \\
Emission-line galaxies (ELG) & 12 & & \\
\hline Total number & 95 & Total number & $42 \mid$ \\
\hline
\end{tabular}



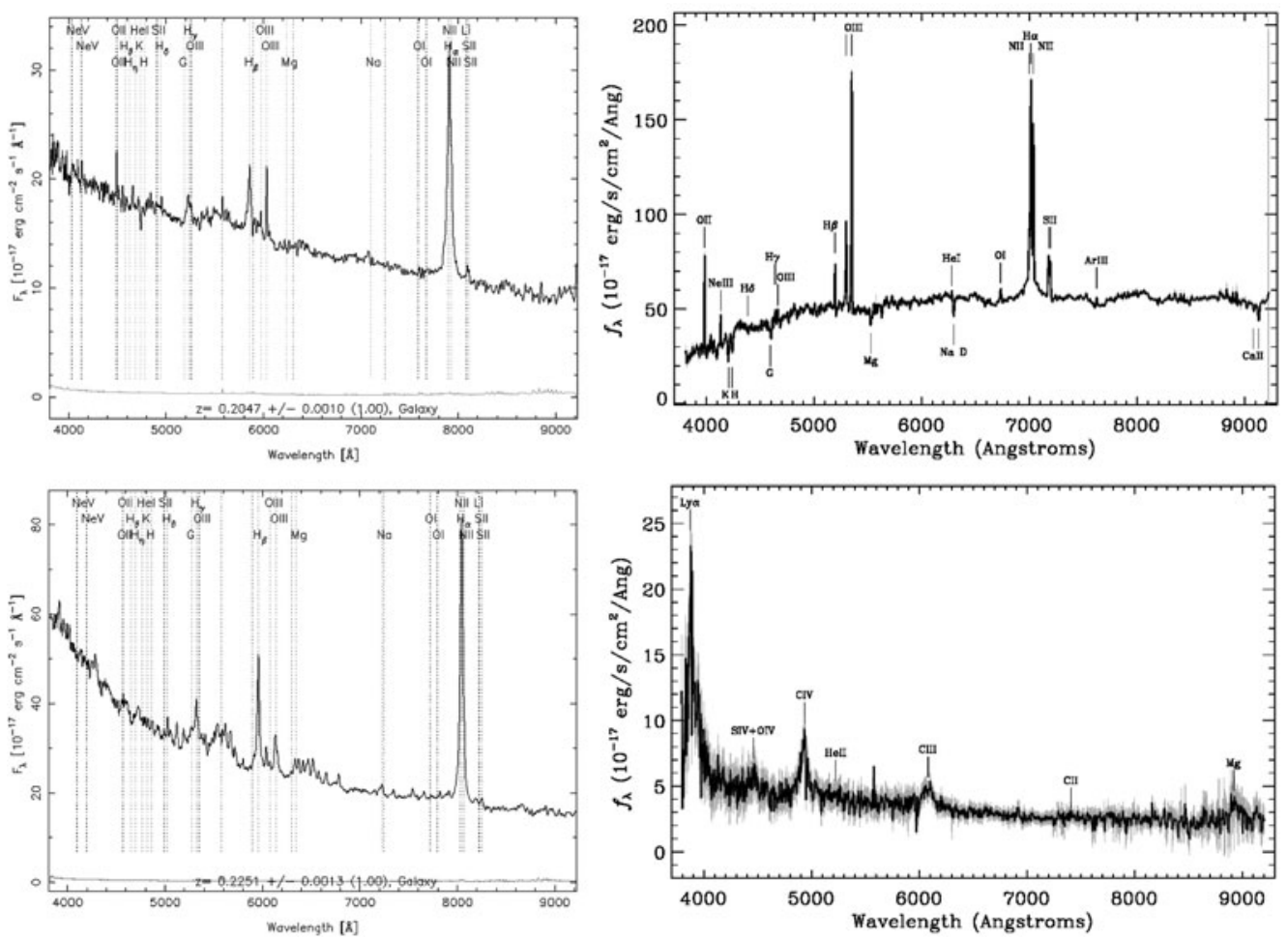

Figure 1. Examples of SDSS spectra classified as AGN: S1.2 (top left), S1.9 (top right), NLS1.5 (bottom left), and QSO (bottom right).

The classification led to the following results: 95 genuine AGN, 71 absorption-line galaxies, 42 stars, and 9 unclassified objects.

Examples of SDSS spectra classified as AGN: S1.2 (top left), S1.9 (top right), NLS1.5 (bottom left), and QSO (bottom right) are given in Fig. 1. The results of the spectral classification for all SDSS DR10 spectra are given in Table 1.

We are going also to carry out detailed studies of other 738 sources using their multiwavelength data in order to be able to confirm their AGN nature, which will help us to use this method to reveal new AGN candidates.

\section{References}

Ahn, C. P., Alexandroff, R., Allende Prieto, C., et al. 2013, ApJS, in press.

Hagen, H.-J., Groote, D., Engels, D., \& Reimers, D. 1995, A\&AS 111, 195

Massaro E., Giommi P., Leto C., et al. 2009, A\& $A$ 495, 691

Mickaelian A. M., Hovhannisyan L. R., Engels D., Hagen H.-J., \& Voges W. 2006, A $\& A 449$, 425

Paronyan G. M. \& Mickaelian A. M. 2014, MNRAS, in press

Veron-Cetty M. P. \& Veron P. 2010, Astron. Astrophys., 518, A10

Voges W., Aschenbach B., Boller T., et al. 1999, Astron. Astrophys, 349, 389

Voges W., Aschenbach B., Boller Th., et al. 2000, IAU Circ, 7432

Zickgraf F.-J., Engels D., Hagen H.-J., Reimers D., \& Voges W. 2003, A\&A 406, 535 